\title{
A comprehensive review of techniques for natural fibers as reinforcement in composites: preparation, processing and characterization
}

\begin{abstract}
Designing environmentally friendly materials from natural resources represents a great challenge in the last decade. However, the lack of fundamental knowledge in the processing of the raw materials to fabricate the composites structure is still a major challenge for potential applications. Natural fibers extracted from plants are receiving more attention from researchers, scientists and academics due to their use in polymer composites and also their environmentally friendly nature and sustainability. The natural fiber features depend on the preparation and processing of the fibers. Natural plant fibers are extracted either by mechanical retting, dew retting and/or water retting processes. The natural fibers characteristics could be improved by suitable chemicals and surface treatments. This survey proposes a detailed review of the different types of retting processes, chemical and surface treatments and characterization techniques for natural fibers. We summarize major findings from the literature and the treatment effects on the properties of the natural fibers are being highlighted.
\end{abstract}

Keyword: Natural fiber; Extraction method; Chemical treatment; Surface treatment; Characterization 